\title{
Microstructure and Dislocation Analysis of $\mathrm{L1}_{0} \mathrm{FePt}$ for HAMR Media Application
}

Yang Yi, Hu Jiangfeng, Kelvin Cher Kiat Min, Wong Seng Kai, Hnin Yu Yu Ko, Nelson Lim Chee Beng, Serene Ng Lay Geok, and Shi Jianzhong

Data Storage Institute, A*STAR (Agency for Science, Technology and Research), Singapore 117608

L1 $1_{0}$ ordered FePt thin films have very high magnetic anisotropy energy $\left(K_{u}\right)$, which ensures the thermal stability as the bit size and grain size become smaller. The particular property is one of the key reasons that granular $\mathrm{L} 1_{0}$-FePt thin films are considered as the most promising candidate for high-density heat assisted magnetic recording (HAMR), a technology geared for future hard disk drives (HDDs). From application viewpoint, FePt based granular film with small well-isolated columnar grains is required. The microstructure control of the FePt based granular film has been reported [1-3]. In general, grain isolation was achieved by fabricating FePt based composite films with a variety of additives such as $\mathrm{SiO} 2, \mathrm{MgO}, \mathrm{ZrO}, \mathrm{C}$, etc.

In this study, we have investigated the microstructure and dislocations of $\mathrm{L} 1_{0}$-FePt granular film by high-resolution transmission electron microscopy (HRTEM) and correlated the results to the thermal conditions and magnetic properties. The FePt based granular films were prepared by industry compatible sputter system where the duration of the heating and deposition processes were within several seconds. Post annealing and a two-step heating processes were applied to enhance the chemical ordering of FePt, in comparison to the one-step heating process. The M-H loop measurement shows FePt with active thermal annealing has a higher in-plane and out-of-plane coercivity.

The plane-view TEM images of FePt media fabricated by one-step, post annealing and two-step heating were shown in Figures 1(a-c), respectively. The microstructure of the one-step heating sample shows large grain size and lateral interconnection of the grains indicated by arrows. A simple post annealing process doesn’t vary the grain size significantly by comparing Figures 1 (a) and (b). In comparison, the two-step heating sample shows much smaller grain size and better grain isolation, although there are still grains interconnected. The annealing effect is further investigated by looking at the FePt/MgO interface. Figures 2(a-c) shows the cross-sectional HRTEM analysis of the $\mathrm{NiTa} / \mathrm{MgO} / \mathrm{FePt}-\mathrm{C} / \mathrm{FePt}_{-} \mathrm{SiO}_{2}$ fabricated by one-step, post annealing, and two-step heating, respectively. The grain size of FePt is usually significantly larger than $\mathrm{MgO}$ buffer layer, as indicated by dotted line in $\mathrm{MgO}$, which is usually a result of grain segregation. As can be seen in Figure 2(a), with one-step heating, the grain boundary in MgO can largely affect the crystalline quality of FePt by inducing $\{111\}$ plane dislocations originated from FePt/MgO interface. After simple post annealing, FePt/MgO interface is similar to Figure 2(a), while only some dislocation can still be observed in FePt grain. On the contrary, as shown in Figure 2(c), with a two-step active heating process, MgO has been pushed upward into the spacing of FePt grains to become part of spacing oxide, and smaller grains formed with less grain segregation. It's obvious that MgO played as isolation oxide before introducing $\mathrm{SiO} 2$ in the two-step heating sample. The FePt grain is around or less than the size of $\mathrm{MgO}$ and dislocations in FePt by the two-step heating are not significant in most of small grains. 
Inter-layer diffusion was further studied by EDX to understand the thermal annealing effect on $\mathrm{FePt} / \mathrm{MgO}$ interface.

\section{References}

[1] T. O. Seki, Y. K. Takahashi, K. Hono, "Microstructure and magnetic properties of FePt-SiO2 granular films with Ag addition”, J. Appl. Phys., vol. 103, pp. 023910, 2008.

[2] G. Sáfrán, T. Suzuki, K. Ouchi, P. B. Barna, G. Radnóczi, "Nano-structure formation of Fe-Pt perpendicular magnetic recording media co-deposited with $\mathrm{MgO}$, Al2O3 and SiO2 additives”, Thin solid films, vol. 496, pp. 580, 2006.

[3] M. Watanabe, T. Masumoto, et al., "Microstructure and magnetic properties of FePt-Al-O granular thin films”, Appl. Phys. Lett. vol. 76, pp. 3971, 2000.
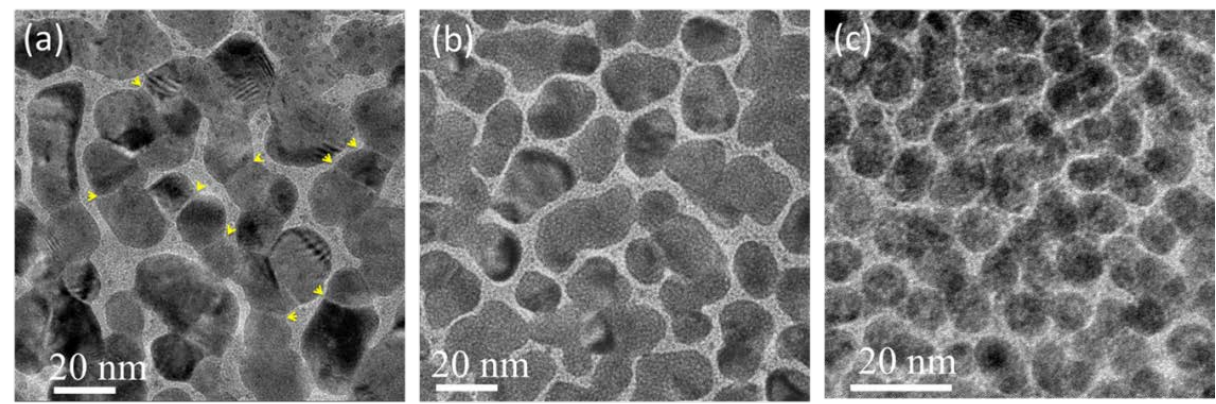

Figure 1 In-plane TEM images of FePt based HAMR media fabricated by (a) one-step heating, (b) post annealing, and (c) two-step heating.
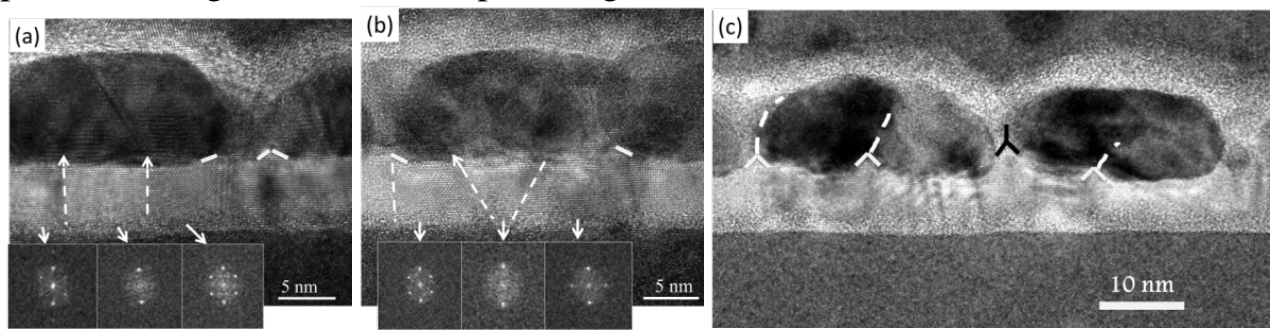

Figure 2. HRTEM analysis of the $\mathrm{NiTa} / \mathrm{MgO} / \mathrm{TiN} / \mathrm{FePt}-\mathrm{C} / \mathrm{FePt}-\mathrm{SiO}_{2}$ fabricated by (a) one-step heating, (b) post annealing, and (c) two-step heating. The MgO spacing oxide induced grain boundaries are indicated in (c). 